\title{
La réglementation du télétravail au Brésil
}

\author{
Juliano Barra
}

\section{(2) OpenEdition}

Journals

Édition électronique

URL : https://journals.openedition.org/rdctss/714

DOI : $10.4000 /$ rdctss. 714

ISSN : 2262-9815

Éditeur

Centre de droit comparé du travail et de la sécurité sociale

Édition imprimée

Date de publication : 1 avril 2021

Pagination : 146-151

ISSN : 2117-4350

\section{Référence électronique}

Juliano Barra, «La réglementation du télétravail au Brésil », Revue de droit comparé du travail et de la sécurité sociale [En ligne], 1 | 2021, mis en ligne le 01 avril 2021, consulté le 01 avril 2022. URL : http:// journals.openedition.org/rdctss/714 ; DOI : https://doi.org/10.4000/rdctss.714

\section{(2) $\odot \Theta \Theta$}

Revue de droit comparé du travail et de la sécurité sociale est mise à disposition selon les termes de la Licence Creative Commons Attribution - Pas d'Utilisation Commerciale - Pas de Modification 4.0 International. 


\section{JULIANO BARRA}

Université Mackenzie-BrésiL, Université PARIS 1 PANTHÉon-SORBONne

\section{LA RÉDUCTION DU SALAIRE EN RAISON DU COVID 19 VALIDÉE PAR LA COUR SUPRÊME DU BRÉSIL}

Face à la pandémie du Covid 19, les gouvernants brésiliens ont pris différentes mesures pour éviter la circulation du virus, privilégiant notamment des restrictions à la libre circulation des personnes. La conséquence directe et immédiate a été la fermeture de plusieurs entreprises dont les commerces et les restaurants, entre autres. L'emploi et l'économie en ont subi de plein fouet les effets néfastes du Covid 19.

Le gouvernement fédéral du Brésil, considérant les chiffres du Ministère de l'Économie, a réagi rapidement et a publié une «Mesure provisoire " (medida provisória) n936 du $1^{\mathrm{er}}$ avril 2020 (par la suite insérée dans la loi $n^{\circ} 14020$ du 6 juillet 2020)1. Cette mesure prévoit la possibilité d'une réduction de la durée du travail et du salaire, voire la suspension du contrat de travail, jusqu'au 31 décembre 2020, par le biais d'accords individuels entre salarié et employeur (I). Un parti politique d'opposition a saisi la Cour Suprême (Supremo Tribunal Federal - STF), estimant inconstitutionnelle la non participation des organisations syndicales à la signature des accords individuels. Par arrêt du 24 novembre de 2020 (action directe en inconstitutionnalité - ADI n. 6363), la Cour suprême a débouté le demandeur et validé la constitutionnalité de la mesure (II).

\section{I - LA PRESTATION D'URGENCE POUR LA PRÉSERVATION DES EMPLOIS ET SALAIRE (BEM)}

La « mesure provisoire " $n^{\circ} 936$ (devenue loi $n^{\circ} 14$ 020) a introduit une " prestation d'urgence pour la préservation des emplois et salaire "(Benefício Emergencial de Preservação da Renda e do Emprego - BEM) versée de façon temporaire pendant la période de réduction de salaire ou de suspension du contrat de travail.

L'objectif du gouvernement fédéral était de préserver le plus grand nombre d'emplois durant cette période déclarée " état d'urgence » (jusqu'au 31/12/2020), et d'essayer

1 "Une "mesure provisoire" (art. 62 C 88) est un acte législatif édicté directement par le Président de la République. Elle acquiert force de loi dès son édiction, sans examen préalable des assemblées, et c'est en ce sens qu'elle est une attribution législative "directe". Son adoption est conditionnée par l'existence de circonstances spécifiques, mentionnées par la Constitution. Seuls les cas d'intérêt particulier et d'urgence justifient sa mise en œuvre. Cependant, l'appréciation de ces circonstances relève de l'entière discrétion du Chef de l'État - le Congrès ne pourra examiner si ces conditions sont réunies qu'après l'entrée en vigueur de l'acte présidentiel. Par ailleurs, la mesure provisoire est par définition temporaire. Dès son adoption, elle est donc immédiatement soumise au vote du Congrès qui, dans un délai déterminé, devra se prononcer sur sa conversion en loi. Si la mesure provisoire n'a pas été appréciée à l'expiration du délai imparti, elle perd son efficacité $a b$ initio à compter de sa publication ». Voir T. Passos Martins, "La Cour suprême du Brésil et l'État démocratique de droit. Contribution à une théorie de la démocratie réflexive », Thèse, Université Montpellier I, 2012, p. 56. 
de maintenir un semblant d'activité économique face à cette réduction d'activité, mais également de réduire l'impact social dû aux conséquences de la pandémie, la « prestation d'urgence " prévue par le gouvernement étant versée en cas de réduction de la durée du travail et du salaire (A) ou en cas de suspension du contrat de travail (B).

\section{A - Réduction DE LA DURÉE DU TRAVAIL ET SALAIRE}

Les employeurs peuvent convenir de réduire proportionnellement la durée de travail et les salaires des salariés, à condition d'observer :

- la préservation du salaire horaire ;

- la durée maximum de l'accord de 180 jours (jusqu'au le 31 décembre 2020 fin de l'état d'urgence);

- I'obligation d'un accord individuel écrit entre l'employeur et le salarié ;

- la garantie provisoire d'emploi pendant la période de réduction et après la reprise du travail pour une durée équivalente à celle de la réduction (exemple : avec une réduction de 2 mois, le salarié aura une garantie d'emploi de 2 mois renouvelable, pour un total de 4 mois, sous peine de versement par l'employeur d'une amende au salarié).

La réduction de la durée de travail proportionnelle à la réduction du salaire a été encadrée par le gouvernement de la façon suivante:

\begin{tabular}{|c|l|l|l|}
\hline Réduction & Valeur de la prestation d'urgence (BEM) & \multicolumn{1}{|c|}{ Accord individuel } & Accord collectif \\
\hline $25 \%$ & $25 \%$ de l'indemnité de l'assurance chômage & Tous les salariés & Tous les salariés \\
\hline $75 \%$ & $75 \%$ de l'indemnité de l'assurance chômage & $\begin{array}{l}\text { Salariés percevant jusqu'à trois } \\
\text { Smic (360€) ou plus de deux } \\
\text { plafonds de la sécurité sociale } \\
(1780 €)\end{array}$ & Tous les salariés \\
\hline $100 \%$ & $100 \%$ de l'indemnité de l'assurance chômage & $\begin{array}{l}\text { Salariés percevant jusqu'à trois } \\
\text { Smic (360€) ou plus de deux } \\
\text { plafonds de la sécurité sociale } \\
(1780 €)\end{array}$ & Tous les salariés \\
\hline
\end{tabular}

Un salarié qui reçoit 2000 Reais (285€) pourra signer un accord individuel avec son employeur pour réduire de $100 \%$ sa durée de travail/salaire, ayant comme résultat le versement par le gouvernement de la prestation d'urgence (BEM) égale à $100 \%$ de l'indemnité de l'assurance chômage. La suspension du contrat de travail a utilisé la même dynamique.

\section{B - LA SUSPENSION DU CONTRAT DE TRAVAIL}

À l'instar de la réduction de la durée de travail/salaire, l'employeur peut négocier la suspension du contrat de travail des salariés, à condition de :

- respecter la durée maximum de l'accord de 180 jours (jusqu'au 31 décembre 2020, fin de l'état de urgence);

- avoir un accord individuel écrit entre l'employeur et le salarié ;

- maintenir, pendant la période de suspension contractuelle, les prestations en nature versées aux salariés (ticket restaurant, mutuelle, etc.) ;

- suspendre totalement le travail y compris en ne lui substituant pas du télétravail pendant toute la durée de la suspension du contrat de travail ; 
- respecter une garantie provisoire d'emploi pendant la période de suspension et après la reprise du travail, pour une durée équivalente à celle de la réduction.

Dès lors, pour qu'un salarié puisse recevoir la prestation BEM et signer l'accord individuellement, il lui reste à observer les conditions suivantes :

\begin{tabular}{|l|l|l|l|l|}
\hline $\begin{array}{l}\text { Chiffre d'affaires annuel } \\
\text { brut de l'entreprise }\end{array}$ & $\begin{array}{l}\text { Aide compensatrice } \\
\text { mensuelle versée par } \\
\text { l'employeur }\end{array}$ & $\begin{array}{l}\text { Valeur de la prestation } \\
\text { d'urgence (BEM) }\end{array}$ & Accord individuel & Accord collectif \\
\hline $\begin{array}{l}\text { Jusqu'à } \\
700000 €\end{array}$ & Non obligatoire & $\begin{array}{l}100 \% \text { de l'indem- } \\
\text { nité de l'assurance } \\
\text { chômage }\end{array}$ & $\begin{array}{l}\text { Salariés percevant } \\
\text { jusqu'à trois Smic } \\
\text { (360€) ou plus de } \\
\text { deux plafonds de } \\
\text { la sécurité sociale } \\
\text { (1780€) }\end{array}$ & Tous les salariés \\
\hline Au-delà de $700000 €$ & $\begin{array}{l}\text { Versement obligatoire } \\
\text { à hauteur 30\% du } \\
\text { salaire du salarié }\end{array}$ & $\begin{array}{l}70 \% \text { de l'indemnité de de } \\
\text { l'assurance chômage }\end{array}$ & $\begin{array}{l}\text { Salariés percevant } \\
\text { jusqu'à trois smic } \\
\text { (360€) ou plus de } \\
\text { deux plafonds de } \\
\text { la sécurité sociale } \\
\text { (1780€) }\end{array}$ & Tous les salariés \\
\hline
\end{tabular}

Même en cas de suspension du contrat ou de réduction de la durée de travail/salaire, l'employeur a l'obligation d'en informer le syndicat dans un délai de 10 jours.

Il existe de nombreuses situations dans lesquelles subsiste la possibilité de réduire la durée de travail et le salaire par le biais d'une simple négociation individuelle. La Cour Suprême a été saisie sur la base de la Constitution la Constitution brésilienne qui interdit théoriquement les réductions de salaire sans négociation collective préalable.

\section{II - LA COUR SUPRÊME VALIDE DES MESURES POUR LE MAINTIEN DES EMPLOIS ET DES SALAIRES PENDANT LA PANDÉMIE}

Le Constituant brésilien a précisé à l'article 2 de la Constitution les relations existant entre les différents pouvoirs : "Les Pouvoirs de I'Union, mutuellement indépendants et en harmonie, sont le Législatif, l'Exécutif et le Judiciaire ». Le Judiciaire est donc un pouvoir indépendant et en harmonie avec les autres. II s'agit d'une conception classiquement présentée depuis Montesquieu, adaptée par la théorie américaine des checks and balances selon laquelle les pouvoirs se contrôlent mutuellement au moyen de freins et de contrepoids (l'équilibre des pouvoirs), et choisie comme modèle politique par le Constituant de 1988. Au Brésil, le droit social dispose d'une assise constitutionnelle forte, au point que le «travailliste » brésilien se doit d'être aussi et avant tout constitutionnaliste.

De fait, la Constitution met un accent tout particulier sur la condition humaine et la valeur sociale du travail, qu'elle décline à travers des principes fondamentaux et des dispositions très précises destinées à régir les rapports professionnels. Les règles en question sont contenues dans le titre relatif aux droits et garanties fondamentaux, et concernent les relations de travail individuelles et collectives. Les relations individuelles font l'objet de l'article 7 consacré aux droits des travailleurs urbains et ruraux, qui comprend 34 paragraphes; les relations collectives se voient quant à elles consacrées dans les articles $8,9,10$ et 11 de la Constitution. 
En dépit de l'initiative du gouvernant d'essayer de réduire le chômage face à la situation d'urgence (avant même la pandémie, le Brésil dénombrait déjà 12,5 millions de chômeurs), un parti d'opposition a saisi la Cour Suprême avec une action directe d'inconstitutionnalité (ADI n. 6363) fondée sur l'argument selon lequel la Constitution interdit la réduction de salaire via un accord individuel, l'autorisant seulement dans le cadre d'une négociation collective, conformément à l'article $7, \mathrm{VI}:$ "La non réduction du salaire, sauf s'il en est disposé autrement par convention ou accord collectif de travail ».

La question serait de déterminer si cet acte juridique (l'accord individuel signé entre salarié et employeur) est passible de révocation par le syndicat, étant donné que l'entreprise est censée l'informer de la signature dudit accord individuel dans un délai de 10 jours, ou si cet acte est parfait et irrévocable, car dénué de tout vice du consentement de la part des salariés. La Cour Suprême a débouté l'auteur de l'ADI sur l'intégralité des motifs présentés. Les juges brésiliens jouent un rôle extrêmement important en matière de droits sociaux, en ce sen qu'ils ont un large pouvoir d'interprétation des dispositifs légaux et constitutionnels. Ce n'est pas sans raison que la doctrine critique, de façon technique et analytique les jugements ayant pour fondements des «principes et valeurs». La Cour constitutionnelle serait actuellement une sorte de «Cour de la proportionnalité et de la raisonnabilité ». Or c'est précisément en s'appuyant sur cette technique que la Cour Suprême a donné une interprétation pondérée des principes de dignité de la personne humaine (art. $\left.1^{\mathrm{er}}\right)^{2}$, de construction d'une société juste et solidaire (art. 3$)^{3}$, du travail comme un droit social (art. $6)^{4}$, et des principes de l'article $7(\mathrm{VI})$ de la Constitution sur la non réduction des salaires.

Par conséquent, les juges de la Cour Suprême brésilienne ont estimé dans l'arrêt du 24 novembre 2020 que la «mesure provisoire » n936 n'avait pas pour seul objectif de légaliser la réduction des salaires, mais plutôt d'établir un mécanisme d'urgence proportionnel but recherché de préserver l'emploi et les salaires. En l'espèce, il ne s'agissait pas d'un conflit entre un salarié et un employeur créant une situation dans laquelle s'imposerait la participation syndicale pour équilibrer les forces en présence. Pour autant, cela ne signifie pas que les syndicats ignorent totalement ces accords puisqu'ils doivent leur être communiqués dans un délai de 10 jours. Ce que le texte légal ne prévoyait pas, c'était d'exiger un avis favorable du syndicat pour que l'accord devienne un acte juridique parfait, ce qui réduirait considérablement l'efficacité de la mesure d'urgence. Ainsi, les juges, à la majorité, ont maintenu la possibilité de réduire la durée du travail/et du salaire ou de suspendre le contrat de travail par le biais des accords individuels, en raison de l'état d'urgence et du contexte particulier de santé publique et de l'économie nationale.

Cette mesure prise par le gouvernement brésilien figure parmi les plus réussies au monde étant donné que ce programme a permis de maintenir plus de 10 millions d'emplois entre avril 2020 et décembre 2020 ; le gouvernement étant actuellement en train d'étudier son extension jusqu'à juillet 2021.

2 «Art. 2 : La République fédérative du Brésil, formée de l'union indissoluble des États, des Communes et du District fédéral, constitue un État démocratique de Droit et a pour fondements: (...) III - la dignité de la personne humaine».

3 «Art. 3. Les objectifs fondamentaux de la République fédérative du Brésil sont les suivants: I construire une société libre, juste et solidaire ».

4 «Art. 6. Sont des droits sociaux l'éducation, la santé, l'alimentation, le travail, le logement, le loisir, la sûreté, la prévoyance sociale, la protection de la maternité et de l'enfance, l'assistance aux indigents, selon les termes de la présente Constitution ». 